\title{
Simulating the effects of thermoelastic deformation on the THESEUS Soft X-ray Imager optics
}

Svendsen, S.; Knudsen, E. B.; Blake, S.; Oosterbroek, T.; Jegers, A. S.; Ferreira, D. D. M.;

Prod'hommec, T. ; Shortt, B.; Willingale, R.; O'Brien, P.

Published in:

Proceedings of SPIE 11119, Optics for EUV, X-Ray, and Gamma-Ray Astronomy IX

Link to article, DOI:

$10.1117 / 12.2529113$

Publication date:

2019

Document Version

Publisher's PDF, also known as Version of record

Link back to DTU Orbit

Citation (APA):

Svendsen, S., Knudsen, E. B., Blake, S., Oosterbroek, T., Jegers, A. S., Ferreira, D. D. M., Prod'hommec, T., Shortt, B., Willingale, R., \& O'Brien, P. (2019). Simulating the effects of thermoelastic deformation on the THESEUS Soft X-ray Imager optics. In S. L. O'Dell, \& G. Pareschi (Eds.), Proceedings of SPIE 11119, Optics for EUV, X-Ray, and Gamma-Ray Astronomy IX (Vol. 11119). [111191R] SPIE - International Society for Optical Engineering. Proceedings of SPIE - The International Society for Optical Engineering https://doi.org/10.1117/12.2529113

\section{General rights}

Copyright and moral rights for the publications made accessible in the public portal are retained by the authors and/or other copyright owners and it is a condition of accessing publications that users recognise and abide by the legal requirements associated with these rights.

- Users may download and print one copy of any publication from the public portal for the purpose of private study or research.

- You may not further distribute the material or use it for any profit-making activity or commercial gain

- You may freely distribute the URL identifying the publication in the public portal 


\section{Simulating the effects of thermoelastic deformation on the THESEUS Soft X-ray Imager optics}

S. Svendsen, E. B. Knudsen, S. Blake, T. Oosterbroek, A. 'S Jegers, et al.

S. Svendsen, E. B. Knudsen, S. Blake, T. Oosterbroek, A. 'S Jegers, D. D. M. Ferreira, T. Prod'homme, B. Shortt, R. Willingale, P. O'Brien, "Simulating the effects of thermoelastic deformation on the THESEUS Soft X-ray Imager optics," Proc. SPIE 11119, Optics for EUV, X-Ray, and Gamma-Ray Astronomy IX, 111191R (9 September 2019); doi: 10.1117/12.2529113

Event: SPIE Optical Engineering + Applications, 2019, San Diego, California, United States 


\title{
Simulating the effects of thermoelastic deformation on the THESEUS Soft X-ray Imager optics
}

\author{
S. Svendsen ${ }^{a}$, E. B. Knudsen ${ }^{b}$, S. Blake ${ }^{c}$, T. Oosterbroek ${ }^{c}$, A. 'S Jegers ${ }^{a}$, D. D. M. Ferreira ${ }^{a}$, \\ T. Prod'homme ${ }^{c}$, B. Shortt ${ }^{c}$, R. Willingale ${ }^{d}$, P. O'Brien ${ }^{d}$

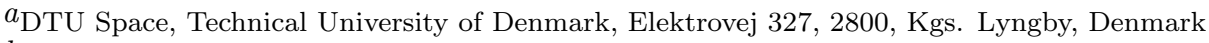

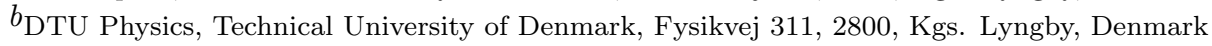 \\ ${ }^{c}$ European Space Agency (ESTEC), Keplerlaan 1, PO Box 299, 2200 AG, Noordwijk, The Netherlands

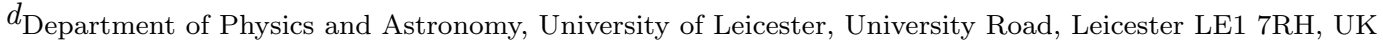

\begin{abstract}
We present a ray tracing simulation of the Soft X-ray Imager (SXI) optics for the THESEUS observatory using the open-source McXtrace software package. THESEUS is a candidate mission within the Cosmic Vision programme of the European Space Agency and is designed to monitor high-energy transient events in the entire sky, therefore relying on fast slewing of the spacecraft. This capability will however impose large temperature variations and gradients across the optics which can cause thermoelastic deformation of the Soft X-ray Imager microchannel optics. The purpose of this study is to evaluate the impact on the point spread function (PSF) due to in-orbit temperature variation. Based on thermal analysis of the Soft X-ray Imager, perturbations to the ideal microchannel geometry are derived and provided as input to the ray tracer.
\end{abstract}

Keywords: THESEUS, Soft X-ray Imager, McXtrace, ray tracing, microchannel optics, lobster eye optics, X-ray optics

\section{INTRODUCTION}

The Transient High Energy Sky and Early Universe Surveyor (THESEUS) mission is one of three candidates proposed for the M5 call within the Cosmic Vision programme of the European Space Agency (ESA). The spacecraft will carry scientific instruments designed to provide a complete survey of the gamma-ray burst population in order to investigate the early universe and to observe transient phenomena throughout cosmic history. Furthermore, THESEUS will play a key role in the next generation of multimessenger observatories ${ }^{1-3}$.

The Soft X-ray Imager (SXI) for the THESEUS spacecraft is designed as a discovery instrument in the soft X-ray band (0.3-6.0 keV), providing a source location accuracy of $<1-2 \operatorname{arcmin}^{4}$. The instrument consists of 4 lobster eye telescopes to form a low-mass X-ray focusing optic that covers a combined field of view of $\sim 1 \mathrm{sr}$. The optical elements consists of microchannels in a closely packed array to increase the collecting area.

Focusing optics for X-ray applications work by means of total external reflection at grazing incidence angles. This makes the lobster eye ideal for X-ray monitoring since constructing large-aperture grazing incidence optics, such as Wolter focusing optics, usually imposes a significant trade-off with the spacecraft mass budget.

The microchannel optics fabrication has reached a high technology readiness level (TRL) owing to the technological advancement in refining channel alignment, channel wall roughnesses and metallization of the channel walls to improve the telescope throughput. The lobster eye optics technology is used for X-ray focusing instruments on current and future missions such as BepiColombo, Einstein Probe, SVOM and SMILE ${ }^{5-8}$.

While ray tracing simulations of lobster eye optics have previously been performed ${ }^{9-12}$, the McXtrace simulation provides a framework to study the effects of thermoelastic deformations of individual channels, applied to a geometry that is representative of the THESEUS SXI instrument. The glass optical elements will deform with the thermal variations of the optic support frame as the spacecraft environment changes. Based on previous thermal tests of the SVOM MXT optics which indicated that a $\Delta T$ of up to $\pm 20 \mathrm{~K}$ from the assembly temperature lead to a decrease in angular resolution, it was recommended to implement thermal control of the MCP optics ${ }^{13}$.

Further author information - send correspondence to:

Sara Svendsen, e-mail: sasv@space.dtu.dk

Optics for EUV, X-Ray, and Gamma-Ray Astronomy IX, edited by Stephen L. O'Dell, Giovanni Pareschi, Proc. of SPIE Vol. 11119, 111191R - (c) 2019 SPIE · CCC code: 0277-786X/19/\$21 - doi: 10.1117/12.2529113 
The exact orbit of THESEUS is still unknown so a set of representative thermal load cases were assumed for the simulations. The purpose of this study is to provide the assumed thermal environments, both steady state and operational gradients, as boundary conditions for the thermoelastic analysis. The resulting deformation map is implemented in the ray tracing in order to assess the requirement for in-orbit thermal control of the SXI optics.

\section{PROPERTIES OF THE SXI MICROCHANNELS}

Inspired by the focusing principles of lobster eyes to provide imaging capability free of the chromatic aberrations of refractive optics and with a large field of view, the SXI optical elements comprise millions of square cross section microchannels placed on a spherical surface ${ }^{14,15}$. The complete optic of an SXI module consists of an $8 \times 8$ array of MCPs, spanning an area of $1332 \mathrm{~cm}^{2}$ excluding the envelope of the support frame. The channels are manufactured from drawn, hollowed square glass fibers fused together in a square packing pattern into thin, square microchannel plates ${ }^{9}$. The MCPs measure $40 \times 40 \mathrm{~mm}^{2}$ and have a plate thickness (microchannel length) of $l=2.4 \mathrm{~mm}$, optimized such that $l / d=60$. The individual $d=40 \mu \mathrm{m}$ square channels are arranged in the MCPs with a $p=52 \mu \mathrm{m}$ pitch.

Each MCP is heat slumped to obtain a spherical profile with a radius of curvature $R=2 \times F_{L}=0.6 \mathrm{~m}$. Figure 1 shows this geometry where the channel axes point radially toward the center of the sphere to focus rays onto a focal plane at half the sphere radius, $F_{L}$.

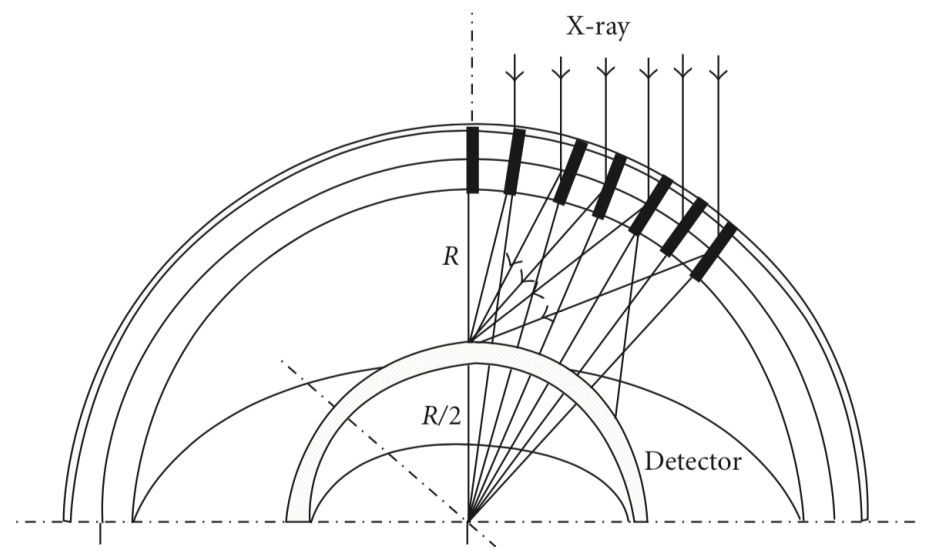

Figure 1: Illustration of the focusing principle of the lobster eye optics geometry $^{15}$.

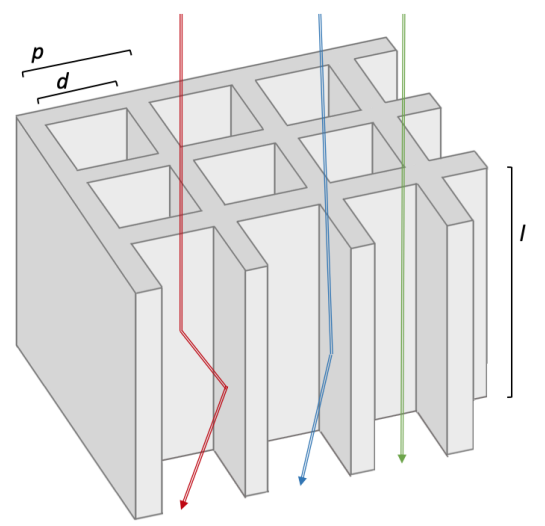

Figure 2: Section a microchannel plate showing the types of reflections.

Incoming X-rays will be focused through a number of reflections off the walls within a channel as shown in Figure 2. The walls will be coated with a $10 \mathrm{~nm}$ Ir film in order to improve the reflectivity within the operational energy range of the telescope. Rays passing through the channels without being reflected (green arrow) give rise to a diffuse background. An even number of wall reflections (red arrow) are focused in the central spot while an odd number of reflections (blue arrow) produces the characteristic cross arm features. However, only rays that reflect off orthogonal walls are focused properly onto the focal plane - rays reflecting off opposite walls contribute to the diffuse background, and the effect generally increases for higher order reflections.

\section{RAY TRACING WITH MCXTRACE}

McXtrace is an open source software package for Monte Carlo simulation of X-ray beamlines which offers a highly modular and flexible framework to create user-defined instruments and individual components along the beam path $^{16}$. The ray tracing code developed to simulate the THESEUS SXI optics inherits from the AstroX toolbox which is a library of X-ray telescope devices for McXtrace currently under development ${ }^{17}$. Originally written to perform ray tracing of the ATHENA silicon pore optics, the components of AstroX allow for the interaction of rays with individual channels arranged in a user-specified geometry. 
A new component simulating a lobster eye configuration was written in the McXtrace framework. The optics geometry for the SXI optic is implemented by placing the microchannels in square arrays corresponding to the positions of the MCPs mounted onto the support frame as illustrated in Figure 3. The channels are placed on the surface of an $R=0.6 \mathrm{~m}$ sphere with the channel axes pointing radially towards the center of the sphere.

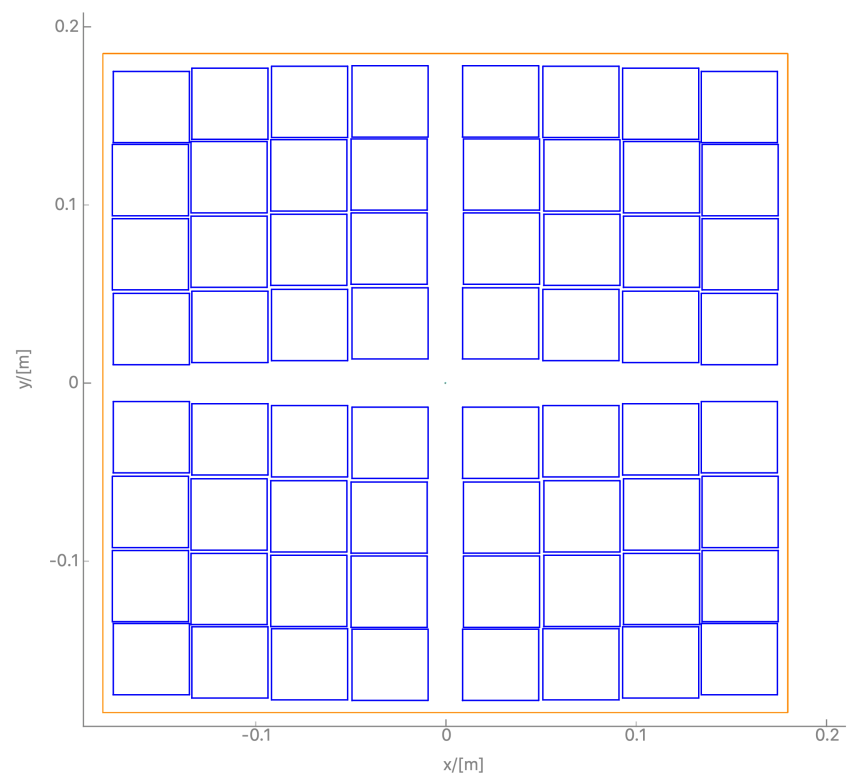

Figure 3: Modelling of the SXI optic in McXtrace showing a front view of the MCP placement. The orange box marks the extent of the source.

The SXI aperture is fully illuminated by an on-axis source emitting a uniform $0.3-6.0 \mathrm{keV}$ spectrum. A 10 mrad beam divergence is imposed on the source placed $10 \mathrm{~m}$ away from the optic. We consider incoming rays that intersect the sphere and hit within the open aperture only. It is assumed that the MCP glass absorbs rays that impact on the wall between channels or the support frame and hence do not enter any channel. Furthermore, no transmission of rays incident at high angles to adjacent channels is permitted.

The energy and incidence angle dependent X-ray reflective properties, $R(\alpha, E)$, of the interior channel walls used to weight the reflectance of rays are simulated using the CXRO optical constants in the IMD software ${ }^{18}$. A 10 $\mathrm{nm}$ Ir coating on a substrate composed of $\mathrm{SiO}_{2}$ with a bulk density of $3.3 \mathrm{~g} / \mathrm{cm}^{3}$ is assumed, representative of the lead-glass used to produce MCPs. The average RMS roughness is fixed to $1.1 \mathrm{~nm}$ based on previous experimental results ${ }^{13}$.

The effects of MCP manufacturing errors are implemented as defects to the individual channel. This includes random channel axis alignment errors which can be introduced during the stacking or slumping processes, causing a misalignment of the channel axis relative to the optics surface normal. Due to the correlation between misalignment errors of adjacent channels, these defects are sampled from a Lorentzian distribution with $\Gamma=$ 1 arcmin. Channel slope errors may occur if the inner walls are not perfectly flat from the etching process hollowing out the channels. These imperfections are therefore approximated as a random change in the slope of the channel wall at each reflection point sampled uniformly within a maximum tilt angle of $\pm 10^{-4} \operatorname{rad}^{19}$.

The simulated deformations are interpolated to each individual channel coordinate and are implemented as displacements to the axis orientation. When a ray intersects the area on the sphere spanned by the SXI, the ID of the channel that would have been hit in the unperturbed case is used to look up the positions of the nearest translated channels, and the actual hit channel is found. The derived rotation of the corresponding hit channel are then implemented by rotating the incoming ray as it passes through the channel. The angular resolution (FWHM of the central spot) is intrinsically limited by the geometric channel size, diffraction and random channel manufacturing errors, and therefore the thermoelastic deformations imposed on the optic are expected to limit the angular resolution additionally. 


\section{FINITE ELEMENT ANALYSIS}

The thermoelastic deformations of the SXI optic are evaluated by a finite element method (FEM) analysis carried using the MD Nastran Version 20141 Solver and the FEMAP 11.0.1 pre and post processor ${ }^{20,21}$. The structural items of the SXI module in the FEM are represented by the microchannel plates (optics), microchannel plate mounting frames, SXI instrument module structure, SXI instrument bipods, SXI instrument focal plane structure, and the SXI instrument module support structure.

Mid plane surfaces are created for each of the solid model entities representing the MCPs in the SXI geometric model. These mid plane surfaces are meshed using CQUAD4 shell elements. The element nodes are forced to the surface of a sphere having a radius of curvature of $600 \mathrm{~mm}$ with its vertex at the geometric centre of the focal plane.

In order to properly represent the bending stiffness of the MCPs, the equivalent bending stiffness is calculated from the geometric properties of the MCP. For the MCP, the pore size is given as $20 \mu \mathrm{m}$ and the wall thickness as $6 \mu \mathrm{m}$. The solidity ratio used to compute the bending stiffness $\left(K=12 I / T^{3}\right)$ is therefore calculated as $6 / \sqrt{20^{2}+20^{2}}=0.21$. The material properties of the MCPs are assumed to be those of BK7 glass with an effective density of $165 \mathrm{~kg} / \mathrm{m}^{3}$ based on the solidity ratio. The corners of each MCP are fixed onto the $\mathrm{Al}$ mounting frame, allowing the plates to bend slightly. The MCPs are assumed to be shell elements and the plate bending is calculated based on the porosity of the MCP. The adhesive bonded edges are not represented in this study.
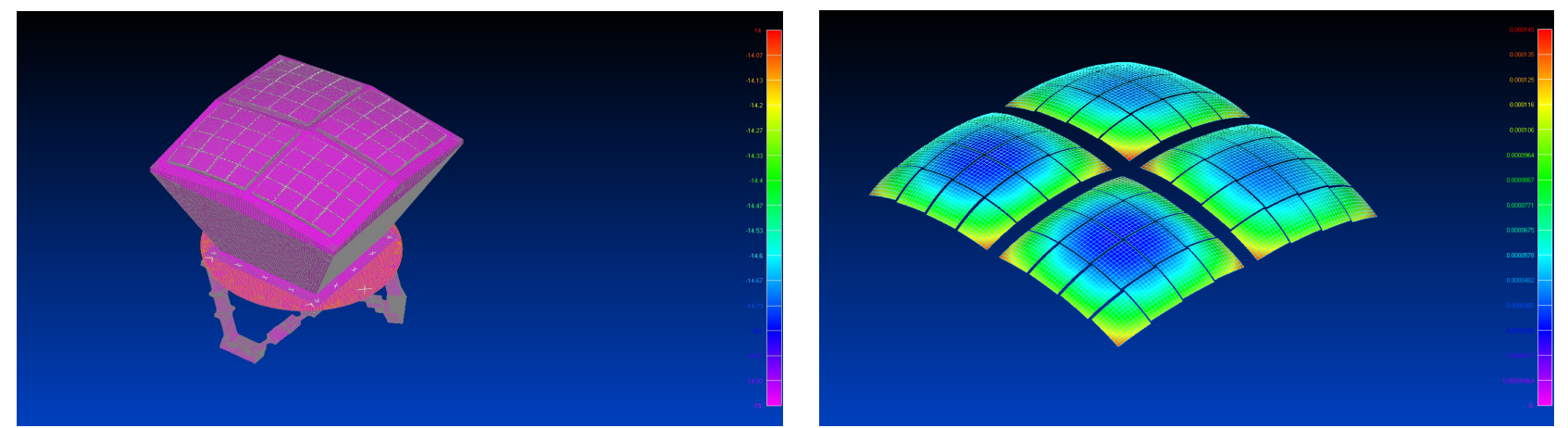

Figure 4: Left: Global temperature offset of $-15 \mathrm{~K}$ applied to the model of SXI module. Right: Degree of thermoelastic deformation of the MCPs as a result of the $-15 \mathrm{~K}$ offset.

Each MCP is modelled with a $10 \times 10$ element mesh to calculate the displacements. The resulting translations and rotations of the nodal mesh were subsequently interpolated to the full channel grid for the ray tracing implementation. Different thermal load case (LC) scenarios relative to the ideal optics case are considered for the simulation:

- LC0: Ideal optics scenario at reference temperature $T_{0}:=273 \mathrm{~K}$,

- LC1: Global temperature load: $\Delta T=-15 \mathrm{~K}$,

- LC2: Global temperature load: $\Delta T=+15 \mathrm{~K}$,

- LC3: Thermal gradient along $x$-axis: $\Delta T=+10 \mathrm{~K} / \mathrm{m}$,

- LC4: Thermal gradient along $y$-axis: $\Delta T=+10 \mathrm{~K} / \mathrm{m}$,

- LC5: Thermal gradient along $z$-axis: $\Delta T=+10 \mathrm{~K} / \mathrm{m}$.

LC0 is intended as the ideal baseline in which the SXI module is thermally controlled to a fixed reference temperature, $T_{0}=273 \mathrm{~K}$. The LC1 and LC2 apply a global temperature offset of $\mp 15 \mathrm{~K}$ relative to LC0, as illustrated in Figure 4.

The temperature gradient case shown in Figure 5 is specified as a $10 \mathrm{~K} / \mathrm{m}$ thermal gradient along each of the three axes. Assuming a worst case scenario where one of side of the SXI is cold and the opposite side warm, the dimensions of the SXI module $(0.4 \mathrm{~m}$ across $)$ results in a total $\Delta T$ of $4 \mathrm{~K}$ in the $x$-and $y$-directions. 

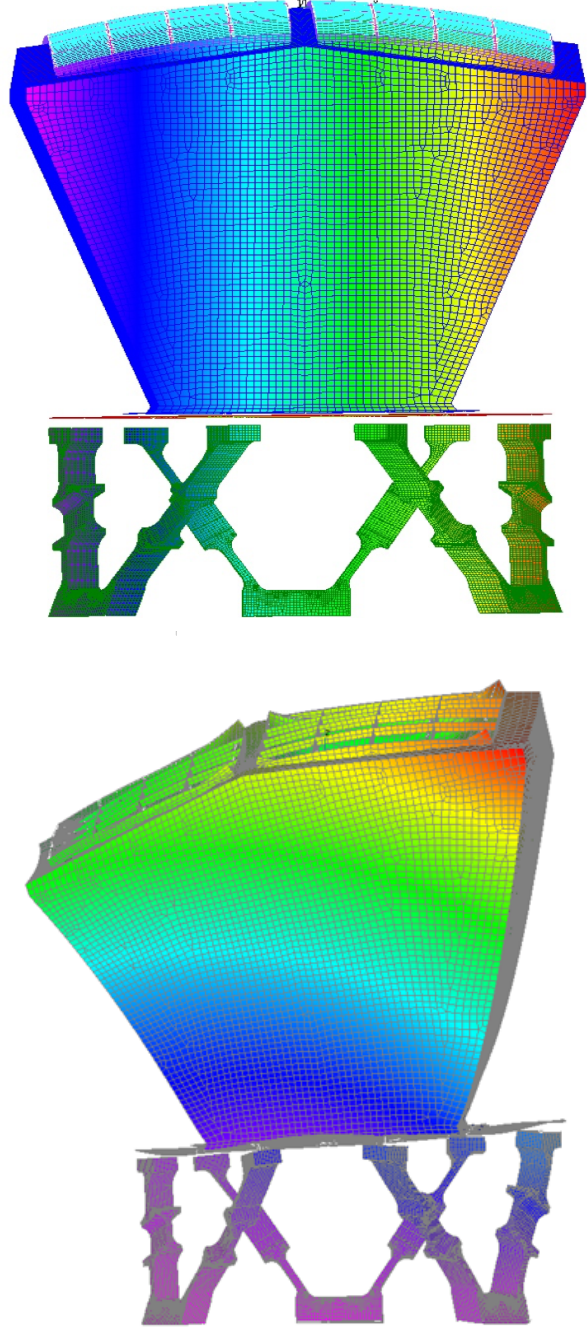
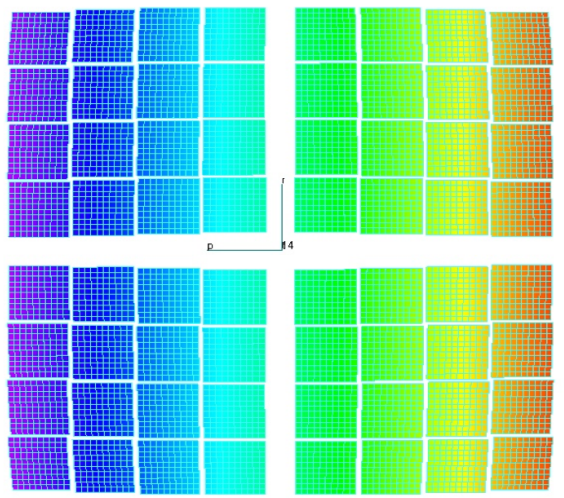

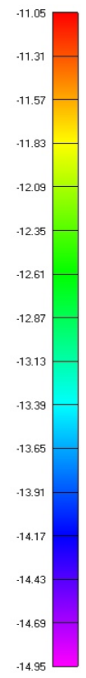
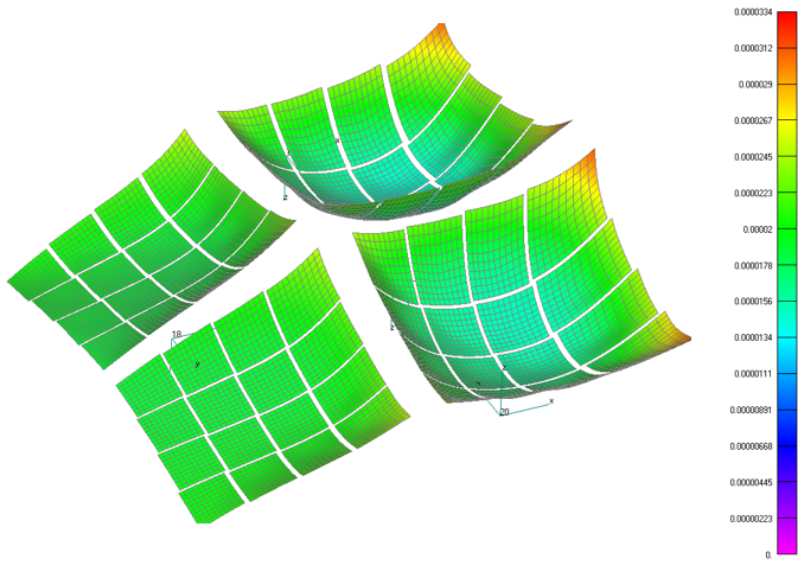

Figure 5: The LC3 thermal gradient of $10 \mathrm{~K} / \mathrm{m}$ applied to the SXI model along the $x$-axis (top) and the amplified, resulting thermoelastic displacements (bottom).

\section{RESULTS}

The PSFs of the SXI ray tracings performed for different thermal load cases and normalized to LC0 are compared in Figure 6 for the global load cases, and in Figure 7 for the gradient cases. All simulation runs take random channel axis misalignment and figure error into account and applies the thermoelastic deformations to each individual channel.

The observed separation between the central focus and line focus cross-arms is produced by the the geometry of the support frame which separates the optic into four quadrants. This is also what gives rise to the asymmetry between the horisontal and vertical line focuses.

Due to the small scale of the deformations, no effect on the image is observed. In all cases, the measured FWHM of the central focus is 0.55 arcmin. Figure 8 shows the encircled energy with increasing distance from the center of the image which is not impacted by any of the imposed thermal load cases. The $25 \%$ encircled energy radius is $14.4 \mathrm{~mm}$. 

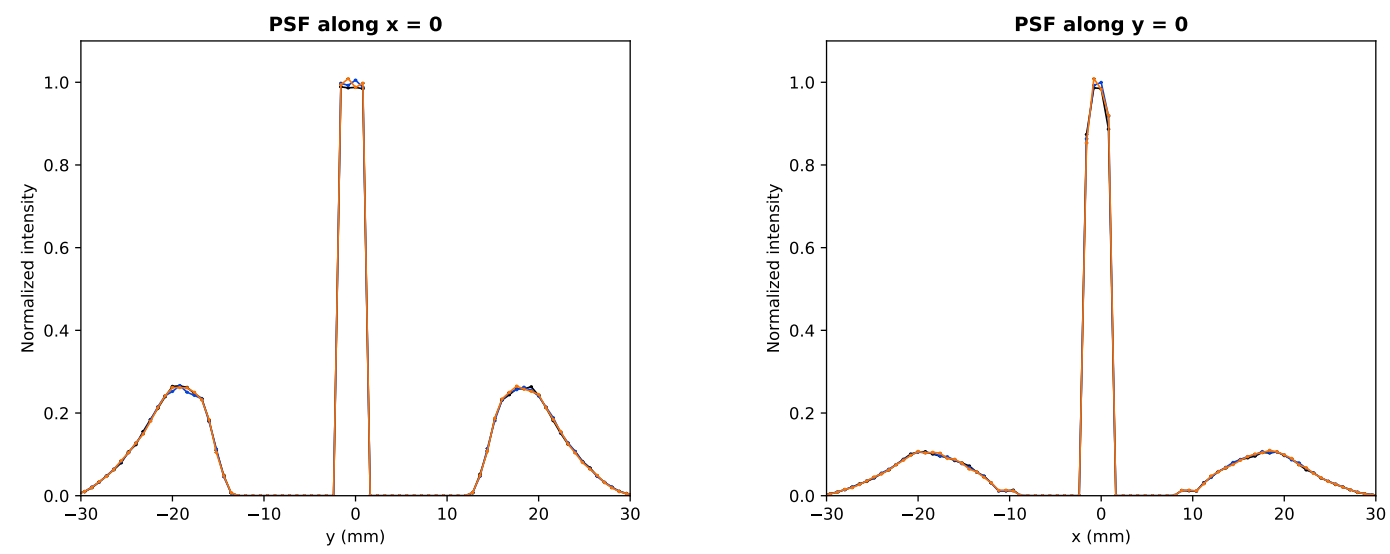

Figure 6: PSF resulting from the ray tracing with global thermal load cases, LC1 and LC2, compared to the reference L0. No deviations between load cases are observed.
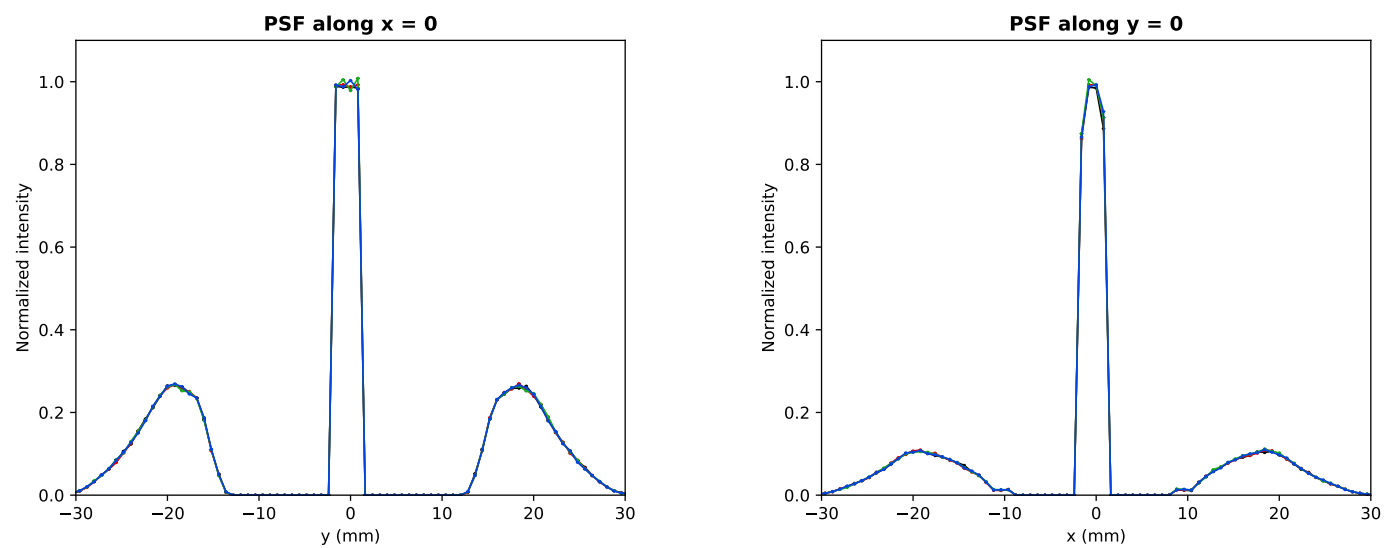

Figure 7: PSF resulting from the ray tracing with gradient thermal load cases, LC3, LC4 and LC5, compared to the reference L0. No deviations between load cases are observed.
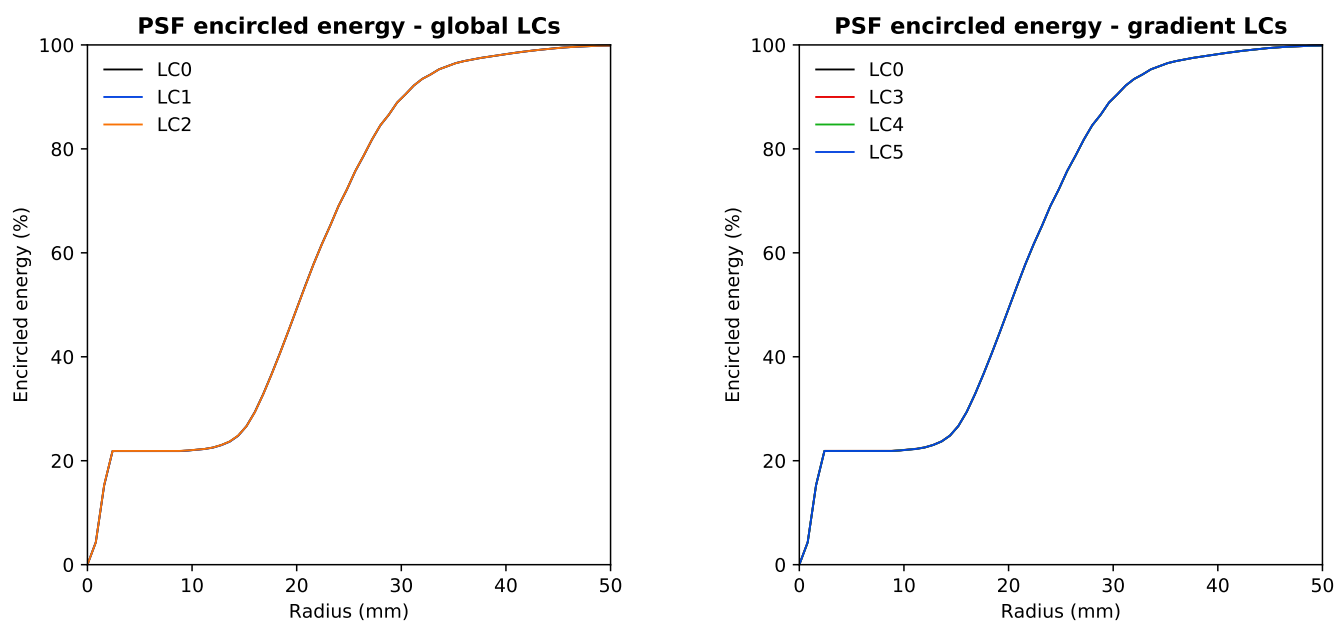

Figure 8: Encircled energy of the PSF for the global (left) and gradient (right) load cases as a function of radius from the center. 
In order to test the potential effects of more severe imposed thermoelastic deformations and to assess to limit to which an image can still be produced on the focal plane of the SXI, the ray tracing was performed with the translations and rotations of the thermal load scaled by factors of 100. Applying higher scale factors to the deformations resulted in a complete degradation of the PSFs.
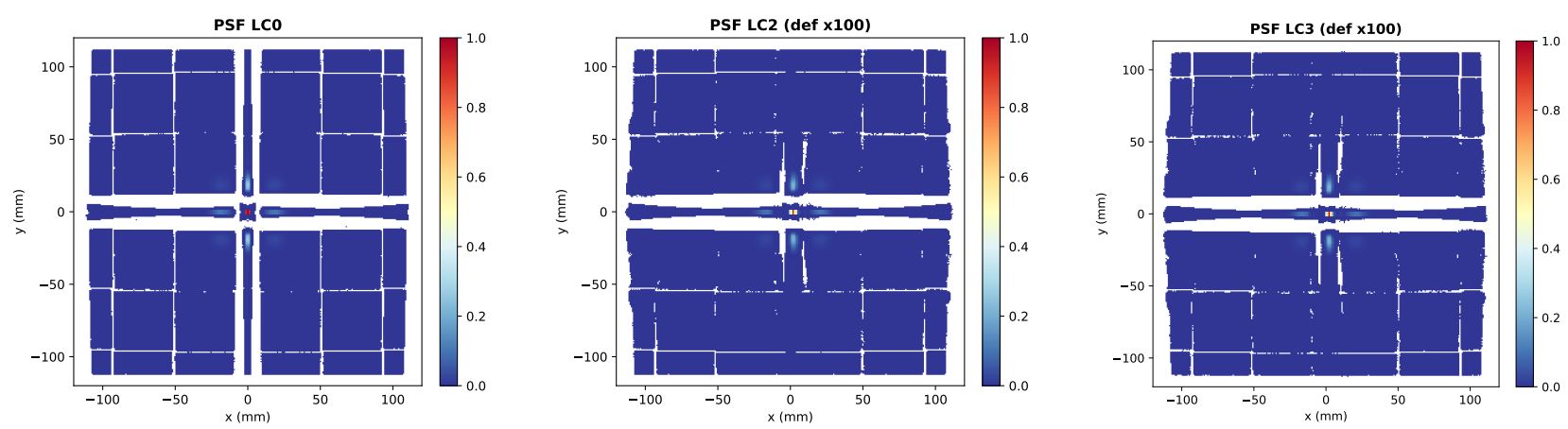

Figure 9: The normalized PSFs of the SXI at the reference LC0 with random manufacturing errors (left), with deformations of LC2 ×100 (center), and deformations of LC3 ×100 (right).

The PSFs for the scaled LC2 and LC3 deformation cases are shown in Figure 9. The gaps (white areas) in the diffuse background surrounding the focus are caused by shadowing by the space between the open aperture of each MCP. In both cases, the scaled-up deformations of the imposed thermal loads largely preserves the central focus and cross-arm features. The dominant effects are the shift and broadening of the central focus along the $x$-axis on the focal plane, as well as the diffusion of the cross-arm signals. In the $y$-direction, the defects mainly cause a loss in intensity of both central and cross-arm focuses. These distortions are a result of the dominant thermoelastic deformation of the SXI module structure (see Figure 5), rather than the deformations of the MCP elements. The reason for the different thermoelastic displacements is due to the bipod configuration which is slightly stiffer along the SXI $x$-axis than along the $y$-axis.
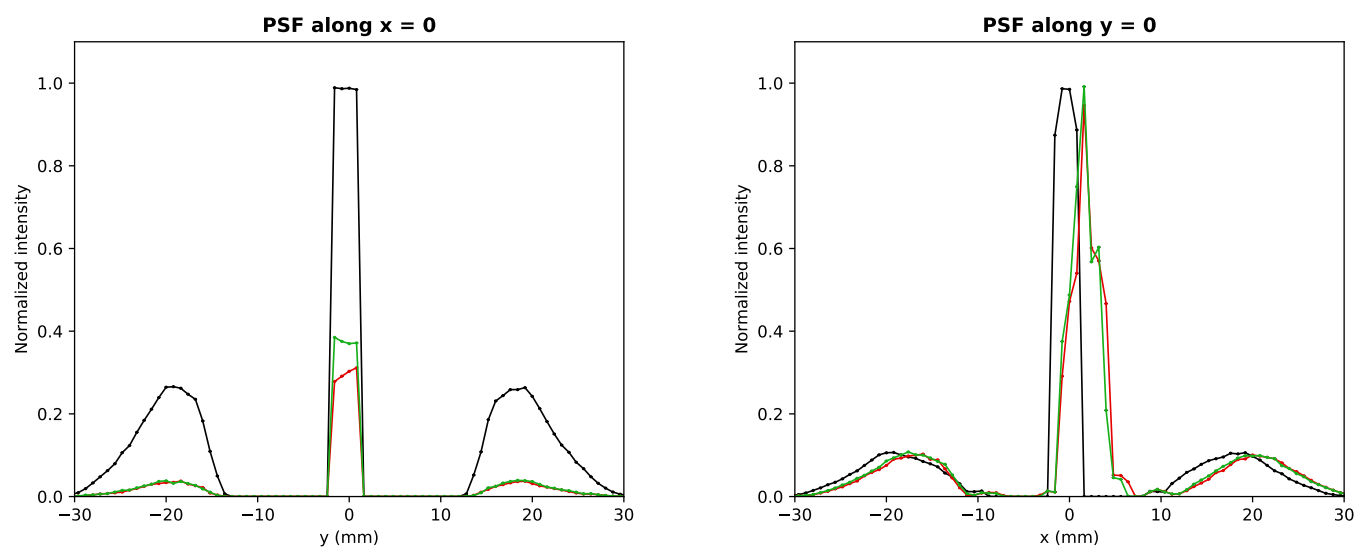

Figure 10: Comparison of the scaled thermal load case PSFs to LC0. 


\section{SUMMARY}

A ray tracing simulation of the THESEUS SXI instrument was performed in the McXtrace framework. The written SXI component is defined such that the positions and orientations of each individual microchannel can be perturbed from the ideal geometry, either by random defects attributed to optics manufacturing errors, or through a deformation map specified in an input file.

An FEM analysis was carried out to evaluate the thermoelastic deformations on the SXI optics for five different realistic thermal load cases. The resulting deformations from the FEM analysis were implemented in the ray tracing simulation, and the impact of thermoelastic performance on imaging performance of the SXI was evaluated. Due to the small scale of the derived channel translations and rotations, no degradation on the PSF was observed for any of the thermal load cases.

Future work on the ray tracing includes evaluating the impact of an extended thermal model based on linear combinations of more representative thermal load cases once the thermal environment of THESEUS is known. As part of including the optic in the McXtrace AstroX toolbox, the component code defining the SXI geometry will be generalized to allow for flexibility in defining placement of MCP elements to simulate various microchannel optic configurations.

\section{REFERENCES}

[1] Amati, L., O'Brien, P. T., Götz, D., and the THESEUS Collaboration, "The Transient High Energy Sky and Early Universe Surveyor (THESEUS)," Proceedings of SPIE 9905 (2016).

[2] Amati, L., O’Brien, P., Götz, D., Bozzo, E., Tenzer, C., Frontera, F., Ghirlanda, G., Labanti, C., Osborne, J. P., and Stratta, G., "The THESEUS space mission concept: science case, design and expected performances," Advances in Space Research 62(1), 191-244 (2018).

[3] Stratta, G., Ciolfi, R., Amati, L., Bozzo, E., Ghirlanda, G., Maiorano, E., Nicastro, L., Rossi, A., Vinciguerra, S., and Frontera, F., "THESEUS: A key space mission concept for Multi-Messenger Astrophysics," Advances in Space Research 62(3), 662-682 (2018).

[4] O'Brien, P., Bozzo, E., Willingale, R., Hutchinson, I., Osborne, J., Amati, L., and Götz, D., "The Soft X-ray Imager (SXI) on-board the THESEUS mission," Mem. S.A.It. 89, 130 (2018).

[5] Fraser, G., Carpenter, J., Rothery, D., et al., "The Mercury Imaging X-ray Spectrometer (MIXS) on BepiColombo," Planetary and Space Science 58(1), 79 - 95 (2010).

[6] Yuan, W., Zhang, C., Feng, H., Zhang, S. N., Ling, Z. X., Zhao, D., Deng, J., Qiu, Y., Osborne, J. P., O'Brien, P., Willingale, R., Lapington, J., Fraser, G. W., and the Einstein Probe team, "Einstein Probe - a small mission to monitor and explore the dynamic X-ray Universe," arXiv e-prints, arXiv:1506.07735 (Jun 2015).

[7] Götz, D., Osborne, J., and MXT Instrument Science Team, "MXT: a light X-ray telescope for X-GammaRay Burst afterglow observations.," Mem. S.A.It. 84, 825 (2013).

[8] Walfried, R., Branduardi-Raymont, G., Wang, C., et al., "SMILE: a joint ESA/CAS mission to investigate the interaction between the solar wind and Earth's magnetosphere," Proceedings of SPIE 9905 (2016).

[9] Willingale, R., Fraser, G. W., Brunton, A. N., and Martin, A. P., "Hard X-ray imaging with microchannel plate optics," Experimental Astronomy 8, 281-296 (1998).

[10] Willingale, R., Fraser, G. W., and Pearson, J. F., "Optimization of square pore optics for the x-ray spectrometer on Bepi-Columbo," Proceedings of SPIE 5900, 319-327 (2005).

[11] Zhao, D., Zhang, C., Yuan, W., Zhang, S., Willingale, R., and Ling, Z., "Geant4 simulations of a wide-angle x-ray focusing telescope," Experimental Astronomy 43(3), 267-283 (2017).

[12] Le Duigou, J. M., Mercier, K., Gonzalez, F., Götz, D., et al., "Optical models of MXT using Zemax," Proceedings of SPIE 10562 (2016).

[13] Feldman, C., Pearson, J., Willingale, R., Sykes, J., Drumm, P., Houghton, P., Bicknell, C., Osborne, J., Martindale, A., O'Brien, P., Fairbend, R., Schyns, E., Petit, S., Roudot, R., Mercier, K., Le Duigou, J.-M., and Götz, D., "Testing and modelling of the SVOM MXT narrow field lobster-eye telescope," Proceedings of SPIE 10399 (2017).

[14] Angel, J. R. P., "Lobster Eyes as X-ray Telescopes," Astrophysical Journal 233 (1979). 
[15] Hudec, R., "Kirkpatrick-Baez (KB) and Lobster Eye (LE) Optics for Astronomical and Laboratory Applications," X-ray Optics and Instrumentation 2010 (2010).

[16] Bergbäck Knudsen, E., Prodi, A., Baltser, J., Thomsen, M., Kjær Willendrup, P., Sanchez del Rio, M., Ferrero, C., Farhi, E., Haldrup, K., Vickery, A., Feidenhans'l, R., Mortensen, K., Meedom Nielsen, M., Friis Poulsen, H., Schmidt, S., and Lefmann, K., "McXtrace: a Monte Carlo software package for simulating X-ray optics, beamlines and experiments," Journal of Applied Crystallography 46, 679-696 (Jun 2013).

[17] Knudsen, E. B., Ferreira, D. D. M., Westergaard, N. J., and et al., "The McXtrace AstroX toolbox: a general ray tracing software package for end to end simulation of X-ray optics for astronomical instrumentation," Proceedings of SPIE 10699 (2018).

[18] Windt, D., "IMD - Software for modeling the optical properties of multilayer films," Computers in Physics 12(4), 360-370 (1998).

[19] Wang, L., Rath, B. K., Gibson, W. M., Kimball, J. C., and MacDonald, C. A., "Performance study of polycapillary optics for hard x rays," Journal of Applied Physics 80, 3628-3638 (1996).

[20] "MSC NASTRAN." www.mscsoftware.com/product/msc-nastran.

[21] "FEMAP software." https://www.plm.automation.siemens.com/global/en/products/simcenter/ femap.html. 